\title{
EasyClone 2.0: expanded toolkit of integrative vectors for stable gene expression in industrial Saccharomyces cerevisiae strains
}

\author{
Vratislav Stovicek $^{1} \cdot$ Gheorghe M. Borja $^{1} \cdot$ Jochen Forster $^{1} \cdot$ Irina Borodina $^{1}$
}

Received: 28 May 2015 / Accepted: 2 September 2015 / Published online: 16 September 2015

(C) The Author(s) 2015. This article is published with open access at Springerlink.com

\begin{abstract}
Saccharomyces cerevisiae is one of the key cell factories for production of chemicals and active pharmaceuticals. For large-scale fermentations, particularly in biorefinery applications, it is desirable to use stress-tolerant industrial strains. However, such strains are less amenable for metabolic engineering than the standard laboratory strains. To enable easy delivery and overexpression of genes in a wide range of industrial $S$. cerevisiae strains, we constructed a set of integrative vectors with long homology arms and dominant selection markers. The vectors integrate into previously validated chromosomal locations via double cross-over and result in homogenous stable expression of the integrated genes, as shown for several unrelated industrial strains. Cre-mediated marker rescue is possible for removing markers positioned on different chromosomes. To demonstrate the applicability of the presented vector set for metabolic engineering of industrial yeast, we constructed xylose-utilizing strains overexpressing xylose isomerase, xylose transporter and five genes of the pentose phosphate pathway.
\end{abstract}

Keywords Industrial yeast - Integrative vectors . Heterologous gene expression - Metabolic engineering · Xylose utilization

Electronic supplementary material The online version of this article (doi:10.1007/s10295-015-1684-8) contains supplementary material, which is available to authorized users.

Irina Borodina

irbo@biosustain.dtu.dk

1 The Novo Nordisk Foundation Center for Biosustainability, Technical University of Denmark, Kogle Allé 6, 2970 Hørsholm, Denmark

\section{Introduction}

The yeast Saccharomyces cerevisiae is an important industrial host for production of bio-based chemicals [27]. The products manufactured by fermentation of natural or genetically engineered $S$. cerevisiae span from fuels (ethanol, isobutanol) and bulk chemicals (succinic acid) to enzymes (invertase) [4, 29] and nutraceutical (resveratrol) [20] and pharmaceutical ingredients (insulin) [28]. For production of high-volume low-cost fuels and chemicals, it is essential that the fermentation process shows high titer, rate and yield of a product on the substrate and thus extensive development of the cell factory is necessary to reach these parameters. Metabolic engineering undertakes a rational approach to redirect the metabolic fluxes towards the desired product by targeted manipulation of the cell's genome involving insertion of heterologous pathways, overexpression and downregulation of multiple genes, construction of synthetic regulatory circuits, etc. [14, 24]. Multiple rounds of genetic engineering are commonly required until the cell with desired properties is obtained. For large-scale industrial applications, robust industrial strains are preferred as the hosts. In biorefineries, yeast cells must perform under various stresses, such as fluctuating temperature and $\mathrm{pH}$, high osmotic pressure and presence of inhibitors coming from biomass hydrolysis [1, 35]. Industrial strains are, however, more difficult to genetically manipulate than the laboratory strains. Such strains are typically prototrophic, diploid, polyploid or even aneuploid and often exhibit low transformation efficiencies and lower levels of homologous recombination [44]. The genetic engineering toolbox for manipulation of industrial strains is currently very limited when compared to the tools for well-studied laboratory strains [2]. 
A widely used way for introduction of heterologous DNA sequence is by the use of autonomously replicating vectors, low copy centromeric and high copy episomal, or integrative vectors [45]. The use of autonomously replicating vectors requires selective pressure and results, especially in case of episomal vectors, in segregational instability and population variation. Thus, the expression of the genes often fluctuates in the cell population [3]. Moreover, when it comes to expression of multiple genes, the maintenance of such vectors in amount of two or more at the same time in single cell is not feasible [11]. If multiple genes are cloned into a single episomal vector, gene loss may occur by homologous recombination [48]. Therefore, chromosomal integration of genetic material is the preferred method for generating stable strains. The ease of homologous recombination in yeast led to the development of cloning-free methods using in vivo assembly of PCR-generated multiple DNA fragments and their insertion into the genome [41]. This can even be facilitated by the use of CRISPR/Cas9 [16] providing a marker-free genome editing tool, which can also be applied for engineering of industrial yeast strains $[37,46]$. However, in vivo assembly is quite error-prone and hence extensive verification using PCR and sequencing must always be performed to check for the correct assembly. Moreover, to ensure the stability of large inserts, the multiple use of homologous sequences (promoters, terminators) should be avoided. Alternatively, yeast integrative vectors allow cloning of the genes and their subsequent integration into the genome [42, 45]. Such vectors enable a non-laborious errorless propagation of the cloned components and their reliable delivery into the cells without the need of laborious genotyping. The integrative vectors do not contain replication origin and they get integrated into a particular genomic location after their delivery into the cell via homologous recombination. To achieve reproducible levels of expression, it is important to select a suitable promoter for driving the expression of a gene [34] and an appropriate genomic location, since the chromatin structure can also influence heterologous gene expression. Traditionally, auxotrophic genes have acted as integration target sites [38, 47]. In addition, various transposable elements and other repetitive chromosomal locations were evaluated in terms of reliable expression $[9,10]$. A series of vectors with various auxotrophic markers, promoters, multiple cloning sites and integration sequences have been developed [9, 15, 38, 47]. Recently, an EasyClone vector set was developed [17] enabling integration by double cross-over events into several intergenic regions, which have been validated for sufficient and reliable expression level and absence of growth impairment, on three different $S$. cerevisiae chromosomes [24]. The insertion sites are interspaced with essential genes voiding risk of chromosomal rearrangements even when the expression cassettes share long sequence homologies [26]. The EasyClone vectors enable cloning of one to two genes with the promoters of choice using convenient uracil-excision based (USER) cloning technique. The vectors offer several selection possibilities via auxotrophic markers and also enable marker recycling via Cre-loxP system [17]. However, they are not suitable for prototrophic strains.

In this work, we present the construction and evaluation of EasyClone 2.0 set of integrative vectors suitable for (over-)expression of (heterologous) genes in both laboratory and industrial $S$. cerevisiae strains. The vectors allow for selection in prototrophic yeast strains as they are combined with six different dominant selection markers. The vectors are based on the EasyClone system, which provides a possibility of cloning of up to two genes, when a bidirectional promoter is used [17] and integration of the construct into well-defined chromosomal locations [26]. We further show that the vector set enables stable integration of three different reporter genes, exhibiting a homogenous expression level in several unrelated industrial strains. The marker genes are flanked with loxP sites allowing for marker rescue and recycling, providing a possibility of repeated rounds of gene insertions. As a proof-of-concept, we introduced a seven-gene xylose consumption pathway into the industrial diploid strains Ethanol Red and CLIB382, the former being used in bioethanol production and the latter is applied as a brewing yeast.

\section{Materials and methods}

\section{Strains and media}

The following $S$. cerevisiae strains were included in this study: prototrophic haploid laboratory CEN. PK113-7D (obtained from Peter Kötter, Johann Wolfgang Goethe University Frankfurt, Germany), diploid industrial bioethanol producer Ethanol Red (obtained from Fermentis A Lesaffre division, France), bioethanol producer CBS7960, isolated from Brazilian ethanol fermentation plants, and an Irish brewer's strain CLIB382 (both strains were obtained from Silas Villas-Bôas, University of Auckland, New Zealand). All the other strains are derivatives of the strains mentioned above and are listed in Table 1. Yeast cells were grown at $30{ }^{\circ} \mathrm{C}$ in standard yeast peptone dextrose (YPD) medium or synthetic complete (SC) medium supplemented with $20 \mathrm{~g} / \mathrm{l}$ agar for preparation of solid medium. For selection, the media were supplemented with $200 \mathrm{mg} / \mathrm{l} \mathrm{G} 418$ sulfate, $200 \mathrm{mg} / \mathrm{l}$ hygromycin $\mathrm{B}, 100 \mathrm{mg} / \mathrm{l}$ nourseothricin or $20 \mathrm{mg} / \mathrm{l}$ phleomycin. For selection on acetamide and D-serine, media containing $0.17 \%$ yeast nitrogen 
Table 1 List of strains constructed in the study

\begin{tabular}{|c|c|c|c|}
\hline Strain name & Parental strain & Description & Integrated vectors \\
\hline CENstCFP & CEN.PK113-7D & $\mathrm{X}-2: \mathrm{TEF} 1 \mathrm{p}-\mathrm{CFP}$ & pCfB2048 \\
\hline CENstRFP & CEN.PK113-7D & X-3:TEF1p-RFP & pCfB2049 \\
\hline CENstYFP & CEN.PK113-7D & X-4:TEF1p-YFP & pCfB2050 \\
\hline CENstFP1 & CEN.PK113-7D & $\begin{array}{l}\text { X-2:TEF1p-CFP } \\
\text { X-3:TEF1p-RFP } \\
\text { X-4:TEF1p-YFP }\end{array}$ & $\begin{array}{l}\text { pCfB2048, pCfB2049, } \\
\text { pCfB2050 }\end{array}$ \\
\hline CENstFP2 & CEN.PK113-7D & $\begin{array}{l}\text { X-2:TEF1p-CFP } \\
\text { X-3:TEF1p-RFP } \\
\text { X-4:TEF1p-YFP marker-less }\end{array}$ & $\begin{array}{l}\text { pCfB2048, pCfB2049, } \\
\text { pCfB2050 }\end{array}$ \\
\hline CENstGFP1 & CEN.PK113-7D & X-2:TEF1p-GFP & pCfB3482 \\
\hline CENstGFP2 & CEN.PK113-7D & X-3:TEF1p-GFP & pCfB3483 \\
\hline CENstGFP3 & CEN.PK113-7D & X-4:TEF1p-GFP & pCfB3484 \\
\hline CENstGFP4 & CEN.PK113-7D & XI-1:TEF1p-GFP & pCfB3485 \\
\hline CENstGFP5 & CEN.PK113-7D & XI-2:TEF1p-GFP & pCfB3486 \\
\hline CENstGFP6 & CEN.PK113-7D & XI-3:TEF1p-GFP & pCfB3487 \\
\hline CENstGFP7 & CEN.PK113-7D & XI-5:TEF1p-GFP & pCfB3488 \\
\hline CENstGFP8 & CEN.PK113-7D & XII-1:TEF1p-GFP & pCfB3489 \\
\hline CENstGFP9 & CEN.PK113-7D & XII-2:TEF1p-GFP & pCfB3490 \\
\hline CENstGFP10 & CEN.PK113-7D & XII-4:TEF1p-GFP & pCfB3491 \\
\hline CENstGFP11 & CEN.PK113-7D & XII-5:TEF1p-GFP & pCfB3492 \\
\hline ERstCFP & Ethanol Red & $\mathrm{X}-2$ :TEF1p-CFP & pCfB2048 \\
\hline ERstRFP & Ethanol Red & X-3:TEF1p-RFP & pCfB2049 \\
\hline ERstYFP & Ethanol Red & X-4:TEF1p-YFP & pCfB2050 \\
\hline ERstFP1 & Ethanol Red & $\begin{array}{l}\text { X-2:TEF1p-CFP } \\
\text { XI-1:TEF1p-YFP } \\
\text { XII-4:TEF1p-RFP }\end{array}$ & $\begin{array}{l}\text { pCfB2048, pCfB2515, } \\
\text { pCfB2516 }\end{array}$ \\
\hline ERstFP2 & Ethanol Red & $\begin{array}{l}\text { X-2:TEF1p-CFP } \\
\text { XI-1:TEF1p-YFP } \\
\text { XII-4:TEF1p-RFP marker- less }\end{array}$ & $\begin{array}{l}\text { pCfB2048, pCfB2515, } \\
\text { pCfB2516 }\end{array}$ \\
\hline ERstFP3 & Ethanol Red & $\begin{array}{l}\text { X-2:TEF1p-CFP } \\
\text { X-3:TEF1p-RFP } \\
\text { X-4:TEF1p-YFP }\end{array}$ & $\begin{array}{l}\text { pCfB2048, pCfB2049, } \\
\text { pCfB2050 }\end{array}$ \\
\hline CBSstFP1 & CBS7960 & $\begin{array}{l}\text { X-2:TEF1p-CFP } \\
\text { XI-1:TEF1p-YFP } \\
\text { XII-4:TEF1p-RFP }\end{array}$ & $\begin{array}{l}\text { pCfB2048, pCfB2515, } \\
\text { pCfB2516 }\end{array}$ \\
\hline CLIBstFP1 & CLIB382 & $\begin{array}{l}\text { X-2:TEF1p-CFP } \\
\text { XI-1:TEF1p-YFP } \\
\text { XII-4:TEF1p-RFP }\end{array}$ & $\begin{array}{l}\text { pCfB2048, pCfB2515, } \\
\text { pCfB2516 }\end{array}$ \\
\hline CENstXYL & CEN.PK113-7D & $\begin{array}{l}\text { §Ty2:TEF1p-CpXylA/TDH3p-PsXYL3 X-2:TEF1p-RPE1/TDH3p-RKI1 } \\
\text { XI-5:TEF1p-PsTAL1/TDH3p-TKL1 } \\
\text { XII-2:TEF1p-CpXylA/TDH3p-PsSUT1 }\end{array}$ & $\begin{array}{l}\text { pCfB2871, pCfB2523, } \\
\text { pCfB2872, pCfB2874 }\end{array}$ \\
\hline ERstXYL & Ethanol Red & $\begin{array}{l}\text { 8Ty2:TEF1p-CpXylA/TDH3p-PsXYL3 X-2:TEF1p-RPE1/TDH3p-RKI1 } \\
\text { XI-5:TEF1p-PsTAL1/TDH3p-TKL1 } \\
\text { XII-2:TEF1p-CpXylA/TDH3p-PsSUT1 }\end{array}$ & $\begin{array}{l}\text { pCfB2871, pCfB2523, } \\
\text { pCfB2872, pCfB2874 }\end{array}$ \\
\hline CLIBstXYL & CLIB382 & $\begin{array}{l}\text { §Ty2:TEF1p-CpXylA/TDH3p-PsXYL3 X-2:TEF1p-RPE1/TDH3p-RKI1 } \\
\text { XI-5:TEF1p-PsTAL1/TDH3p-TKL1 } \\
\text { XII-2:TEF1p-CpXylA/TDH3p-PsSUT1 }\end{array}$ & $\begin{array}{l}\text { pCfB2871, pCfB2523, } \\
\text { pCfB2872, pCfB2874 }\end{array}$ \\
\hline
\end{tabular}

base (without aminoacids and ammonium sulfate), $6.6 \mathrm{~g} / \mathrm{l}$ of potassium sulfate and either $0.6 \mathrm{~g} / 1$ acetamide or $2 \mathrm{~g} / \mathrm{l}$ D-serine, respectively, were used. In particular cases in YPD medium, glucose was replaced by $20 \mathrm{~g} / \mathrm{l}$ xylose (YPX) or
$20 \mathrm{~g} / \mathrm{l}$ galactose (YPGal). E. coli strain DH5 $\alpha$ was used as a host for cloning and plasmid propagation. E. coli cells were grown at $37{ }^{\circ} \mathrm{C}$ in Luria-Bertani (LB) medium containing $100 \mathrm{mg} / \mathrm{l}$ ampicillin or $50 \mathrm{mg} / \mathrm{l}$ kanamycin. 


\section{Plasmid construction}

The new set of integrative vectors (Fig. 1, Supplementary material Table S1, the vector set along with maps and sequences can be obtained from Addgene) was constructed by USER fusion [31]. The particular BioBricks (original vector backbone, the details on construction are provided in Supplementary material Supplementary Methods, and marker gene expression cassettes) (Supplementary material Table S2) were amplified by PCR with PfuX7 polymerase [30] under the following conditions: $98{ }^{\circ} \mathrm{C}$ for $2 \mathrm{~min}, 30$ cycles of $98{ }^{\circ} \mathrm{C}$ for $10 \mathrm{~s}, 54{ }^{\circ} \mathrm{C}$ for $10 \mathrm{~s}, 72{ }^{\circ} \mathrm{C}$ for $30 \mathrm{~s} / 1 \mathrm{~kb}, 72{ }^{\circ} \mathrm{C}$ for $10 \mathrm{~min}$. The used templates and primers are listed in Supplementary material Table S1 and Table S3. All the marker gene cassettes (Fig. 1, Supplementary material Table S2) were synthesized by GeneArt (Life Technologies). DNA fragments were gel purified and incubated in HF buffer (New England BioLabs) together with USER enzyme (New England BioLabs) for $25 \mathrm{~min}$ at $37{ }^{\circ} \mathrm{C}$ followed by incubation at $25{ }^{\circ} \mathrm{C}$ for $25 \mathrm{~min}$. The reactions were transformed into chemically competent $E$. coli cells. For cloning of particular genes, the vectors were digested with FastDigest AsiSI (Life Technologies) restriction endonuclease, nicked with $N b$. BsmI (New England BioLabs) and assembled with PCR amplified genes and promoter(s) of choice by USER reaction and subsequent transformation into $E$. coli. Heterologous genes were either synthesized by GeneArt or amplified from the genomic DNA of $S$. cerevisiae
Fig. 1 New set of EasyClone2.0 vectors with dominant markers. a Schematic illustration of the vector structure and the dominant marker cassettes. b Table of the vectors presenting combinations of particular vector (integration site) with the dominant markers

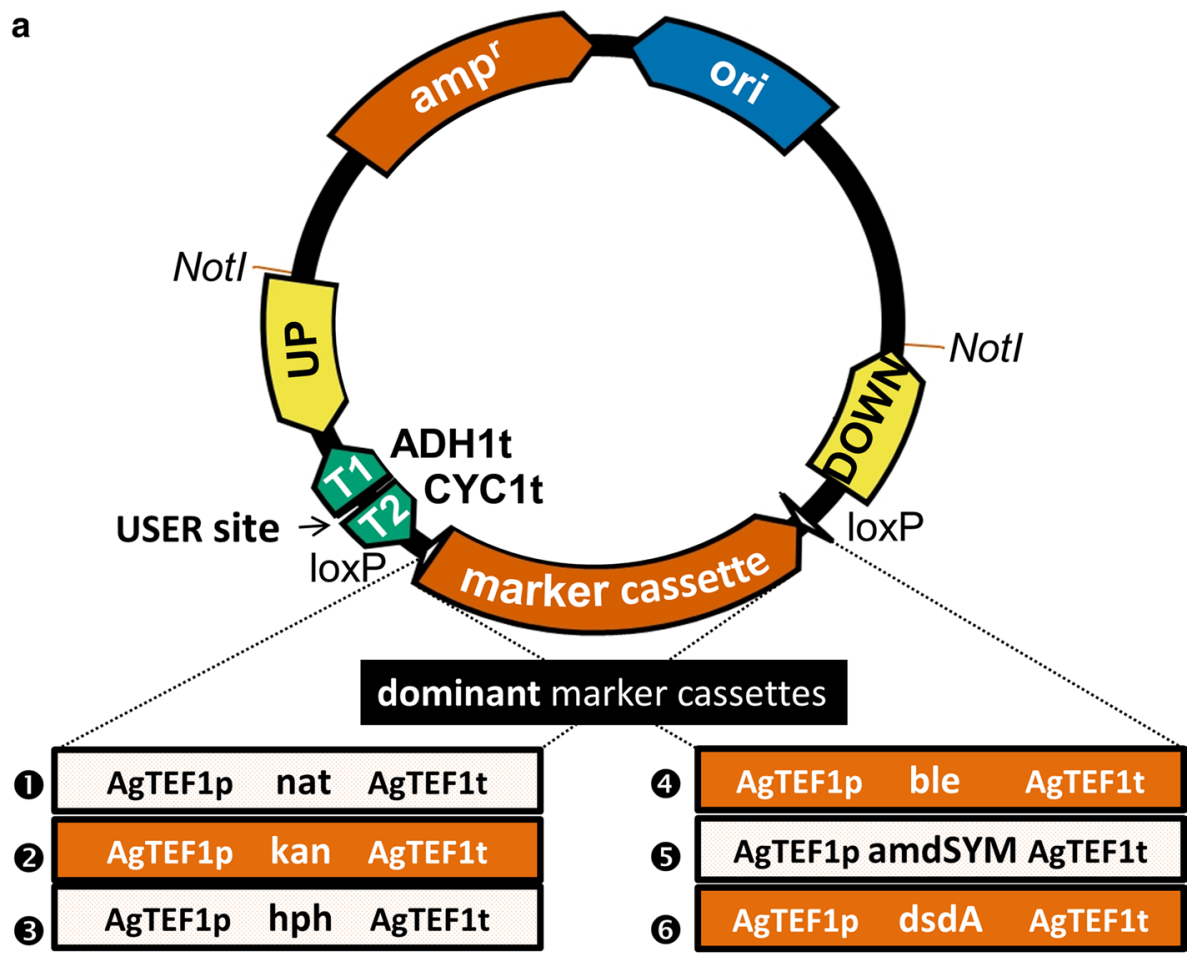

b

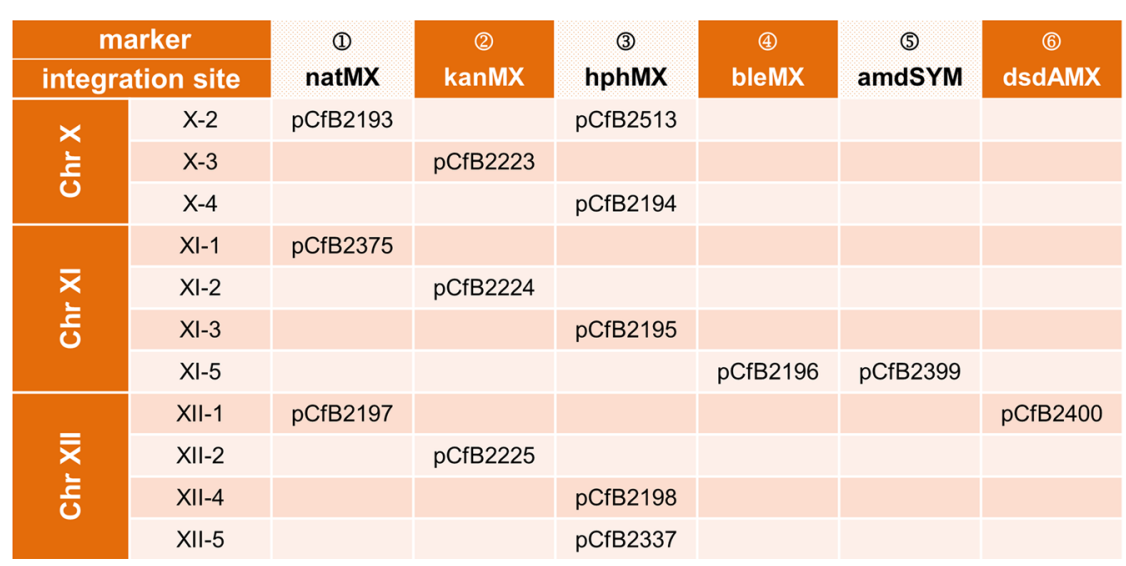


CEN.PK113-7D or Pichia stipitis DSM-3651 (obtained from The Leibniz Institute DSMZ-German Collection of Microorganisms and Cell Cultures) (Supplementary material Table S2). To test the selection properties of the dominant markers, the disruption cassettes for homologous recombination-mediated gene replacement were prepared by PCR [36]. One cassette consisted of the upstream $U R A 3$ region and the first $2 / 3$ part of a particular marker, and the other cassette contained the last $2 / 3$ part of the marker and the downstream sequence of URA3. Both fragments were made by fusion PCR and used for yeast transformation.

\section{Strain construction}

Before yeast transformation, the integrative vectors were linearized by FastDigest NotI (Life Technologies) restriction enzyme. Yeast cells were transformed by PEG/LiAc method according to [12]. The heat shock time was prolonged to $90 \mathrm{~min}$ for strain CBS7960 giving on average 3 -fold higher transformation efficiency in this particular strain. Marker loop-out was performed by transforming the cells with Cre-recombinase carrying centromeric vector (Supplementary material Table S1). The Cre-recombinase expression was induced by cultivation of the yeast cells in YPGal medium for $6 \mathrm{~h}$. After the induction, the cells were plated on non-selective YPD plates. After 2 days, the colonies were replica-plated on selective media containing particular selection agent. The clones lacking the selection marker(s) were verified by colony PCR.

\section{Stability of reporter gene integration}

Yeast strains expressing three reporter genes were grown in three replicates in $1 \mathrm{ml}$ of non-selective YPD medium in deep-well plates at $30{ }^{\circ} \mathrm{C}$ with agitation at $300 \mathrm{rpm}$. Approximately, every $20 \mathrm{~h} 50 \mu \mathrm{l}$ of the culture was transferred into fresh medium. Four serial transfer cycles were performed for a total of approximately 35 generations. The final strains along with the parental strains were analyzed by flow cytometry.

\section{Sensitivity of the strains to selection agents}

The strain sensitivity was tested by the drop assay. The cells were pre-grown in YPD overnight. Subsequently, a water suspension containing $10^{8}$ cells per $\mathrm{ml}$ was prepared. Then, $5 \mu 1$ drops of 10 -fold serial dilutions were applied to YPD plates supplemented with particular antibiotic. Plates were scored after 2 days of growth.

\section{Fluorescence microscopy and fluorescence measurement}

The fluorescent protein expressing cells were observed under $100 \times / 1.25$ immersion oil objective using Leica DM4000 fluorescence microscope. The pictures were captured using Leica DFC300FX digital color camera and LAS 4.0 software. Excitation BP436/12, BP500/20, BP545/40 and suppression filters BP467/37, BP535/30, BP610/75 were used for CFP, YFP and RFP, respectively. Measurement of GFP fluorescence was carried out in standard round 96-well microtiter plates with an optical bottom (Greiner Bio-one, Germany). The fluorescence levels were monitored using Biotek Synergy MX multi-mode plate reader in 485/520 nm emission/excitation wavelength. The sensitivity of the photomultiplier was adjusted to $80 \%$. Overnight cultures of selected clones were twenty times diluted into SC media (with complete supplement mixture) and grown for $6 \mathrm{~h}$ with shaking. GFP fluorescence and $\mathrm{OD}_{600 \mathrm{~nm}}$ of ten times diluted samples were determined.

\section{Flow cytometry}

The cells grown overnight in YPD medium were washed twice with water, resuspended in phosphate-buffered saline buffer and subsequently analyzed on a BD FACSAriaflow cytometer equipped with three solid-state diode lasers: aircooled Coherent ${ }^{\mathrm{TM}}$ Sapphirelaser $(488 \mathrm{~nm}, 100 \mathrm{~mW})$, Yellow Green laser (561 nm, $100 \mathrm{~mW}$ ) and Deep Blue laser $(445 \mathrm{~nm}, 50 \mathrm{~mW})$. The following filters were used: FITCA, PECy5-A, and mCFP-A for the analysis of emission of yellow fluorescent protein (YFP), red fluorescent protein (RFP), and cyan fluorescent protein (CFP), respectively. Compensation was performed according to the BD FACSDiva software protocol. Flow cytometry data sets were subsequently analyzed and interpreted by FlowJo software.

\section{HPLC analysis}

The engineered xylose consuming strains were cultivated in three replicates in $50 \mathrm{ml}$ of YPX medium in shake flasks with constant agitation $(250 \mathrm{rpm})$. Samples were collected at certain time points and the concentration of xylose in culture supernatants was determined by HPLC (UltiMate 3000, Dionex). The samples were analyzed for $30 \mathrm{~min}$ using Aminex HPX-87H ion exclusion column with a $5 \mathrm{mM}$ $\mathrm{H}_{2} \mathrm{SO}_{4}$ flow of $0.6 \mathrm{ml} / \mathrm{min}$. The injection volume was $30 \mu \mathrm{l}$. The temperature of the column was $60{ }^{\circ} \mathrm{C}$. Xylose was detected using RI-101 Refractive Index Detector (Dionex). The refractive index was measured at $45^{\circ} \mathrm{C}$. The data were acquired and analyzed with Chromeleon software. 


\section{Quantitative PCR (qPCR) analysis of gene copy number}

For determination of $C p X y l A$ gene copy number, qPCR analysis was performed using $A L G 9$ as a reference gene. Primers (Supplementary material Table S3) were designed using the PrimerQuest design tool (Integrated DNA Technologies). DNA extraction was carried out using ZR Fungal/Bacterial DNA MicroPrep ${ }^{\mathrm{TM}}$ Kit (Zymo Research). The qPCRs run in three replicates were performed on the Mx3000P qPCR System (Agilent Technologies) using Brilliant III Ultra-Fast QRT-PCR Master Mix (Agilent Technologies), forward and reverse primers (final concentration $200 \mathrm{nM})$, genomic DNA (0.001-50 ng per $20 \mu \mathrm{l}$ reaction) from strains CENstXYL, ERstXYL and CLIBstXYL (or water in negative controls) and $0.3 \mu 1$ per $20 \mu l$ reaction of 1:500 diluted reference dye. An initial melting/activation step at $95{ }^{\circ} \mathrm{C}$ for $10 \mathrm{~min}$ was followed by 40 cycles of melting and annealing/extension $\left(95^{\circ} \mathrm{C}\right.$ for $20 \mathrm{~s}, 60{ }^{\circ} \mathrm{C}$ for $22 \mathrm{~s}$ ) with a fluorescence measurement at the end of each amplification step. Raw data were analyzed using MxPro software (Agilent Technologies). PCR efficiency calculated from the slope of the standard curve was $90 \%$. Ct values of $5 \mathrm{ng}$ dilutions of genomic DNA (providing standard deviation lower than 0.23 ) were used for determination of a gene copy number. The normalized gene copy number was calculated by absolute quantification based on standard curve of the reference gene (assuming 1 copy of the $A L G 9$ per haploid genome) using the following equation: copy number $(C p X y l A)=\frac{\text { copy quantity }(C p X y l A)}{\operatorname{copy} \text { quantity }(A L G 9)}$.

\section{Results}

\section{Construction of a set of EasyClone vectors with dominant selection markers}

To enable the use of the EasyClone system [17] for engineering of industrial yeast strains, we constructed a new set of integrative vectors allowing for selection of a genetic intervention in prototrophic strains (Fig. 1a). For such vectors, an auxotrophic selection cassette in the original set was substituted for one of the six synthetic dominant selection markers (Fig. 1a). Selection genes conferring resistance to several substances (with resistance to antibiotics as the most common) are available. Initially, CEN.PK, Ethanol Red, CBS7960 and CLIB382 strains were tested for their sensitivity to antibiotics G418, nourseothricin, hygromycin B and phleomycin. All the strains grown on YPD were sensitive to the antibiotics in common concentrations as follows: G418-200 mg/l, nourseothricin-100 mg/l, hygromycin B-200 $\mathrm{mg} / \mathrm{l}$ and phleomycin-20 mg/l (Supplementary material Fig. S1). Two other dominant marker gene expression cassettes were used. This involved amdSYM cassette containing acetamidase gene which confers yeast cells the ability to use acetamide as a nitrogen source [43] and dsdAMX containing D-serine deaminase gene which confers yeast cells resistance to D-serine and the ability to use D-serine as a nitrogen source [50]. The ability of all marker gene expression cassettes (containing marker gene, TEF1 promoter and TEF1 terminator from Ashbya gossypii) to confer the resistance was evaluated. Yeast cells were transformed with PCR-generated disruption cassettes carrying the marker genes and selected on corresponding media. Randomly selected transformants were verified for presence of the particular marker cassette. An appearance of neither false positives nor spontaneous resistance mutants was observed. Certain background growth on media containing acetamide or D-serine appeared. However, the positive transformants always got a growth advantage and significantly overgrew the background (not shown).

\section{EasyClone2.0 vectors allow stable integration of multiple genes into industrial strains}

The vectors carrying all eleven insertion sequences (Fig. 1b) were examined and mutually compared in terms of transformation efficiency, correct integration and reporter expression level. To evaluate this, GFP reporter gene under control of constitutive TEF1 promoter was cloned into the vectors carrying dominant markers and the resulting vectors (Supplementary material Table S1) were subsequently transformed into the CEN.PK, Ethanol Red, CLIB382 and CBS7960 cells. The reporter gene carrying vectors were delivered into the CEN.PK strain with comparable transformation efficiency in a range of $1.2 \times 10^{3}$ $3.7 \times 10^{3}$ cells $/ \mu \mathrm{g}$ DNA, with an exception of XI-1 insertion sequence-containing vector that yielded lower amount $\left(6.25 \times 10^{2}\right)$ of transformants (Fig. 2a). The efficiency was not surprisingly lower in case of the industrial strains and differed more among particular vectors being in a range of $1.9 \times 10^{2}-1.8 \times 10^{3}$ cells $/ \mu \mathrm{g}$ DNA in case of Ethanol Red strain, 40-5.3 $\times 10^{2}$ cells/ $\mu$ g DNAin CLIB382 and $2-1.13 \times 10^{2}$ cells/ $\mu \mathrm{g}$ DNA in CBS7960 strain, with the vector XI-1 providing the lowest yield in all cases (Fig. 2a). The vectors integrated at the desired insertion site in the genome of the CEN.PK strain with $80-100 \%$ accuracy (Fig. 2b), as verified by evaluation of randomly selected clones by colony PCR. GFP fluorescent levels were examined under the bench top UV transilluminator revealing homogenous level of the fluorescence. Fluorescence of ten clones of the CEN.PK strain from each vector transformation was determined in microtiter plates by the fluorescence plate reader. The results showed homogenous level of expression among clones from the particular insertion 
Fig. 2 Characteristics of the EasyClone 2.0 vectors. a Transformation efficiency of CEN. PK, Ethanol Red, CLIB382 and CBS7960 strains with the EasyClone 2.0 vectors. Values of the transformation efficiency (expressed as a number of transformants per $\mu \mathrm{g}$ DNA) are plotted on logarithmic scale. The values represent an average from two parallel experiments. Error bars represent standard deviation $(N=2)$. The amount of $10^{8}$ cells was used for each strain and plasmid transformation. b Percentage of correct vector integration as evaluated by colony PCR of ten randomly selected CEN.PK clones. c Relative fluorescence intensity of ten randomly selected CEN. PK clones. Error bars represent standard deviation $(N=10)$

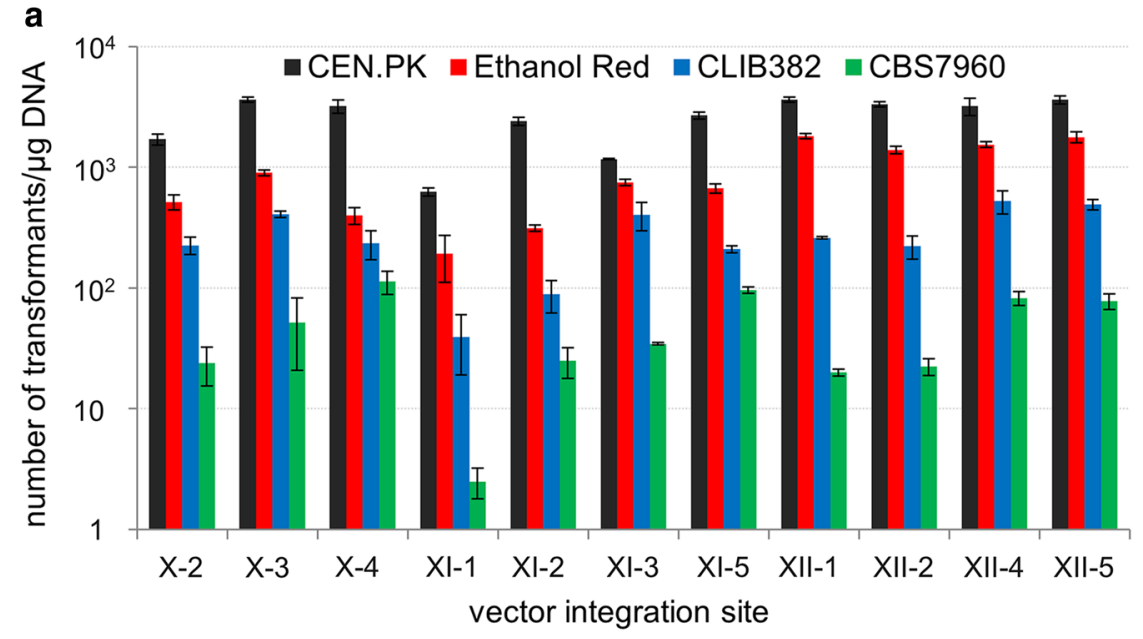

b

\begin{tabular}{lr} 
Correct integration & \multicolumn{1}{c}{$\%$} \\
\hline X-2 & 90 \\
X-3 & 100 \\
X-4 & 100 \\
XI-1 & 100 \\
XI-2 & 100 \\
XI-4 & 100 \\
XII-1 & 100 \\
XII-2 & 100 \\
XII-3 & 100 \\
XII-4 & 80 \\
XII-5 & 100 \\
\hline
\end{tabular}

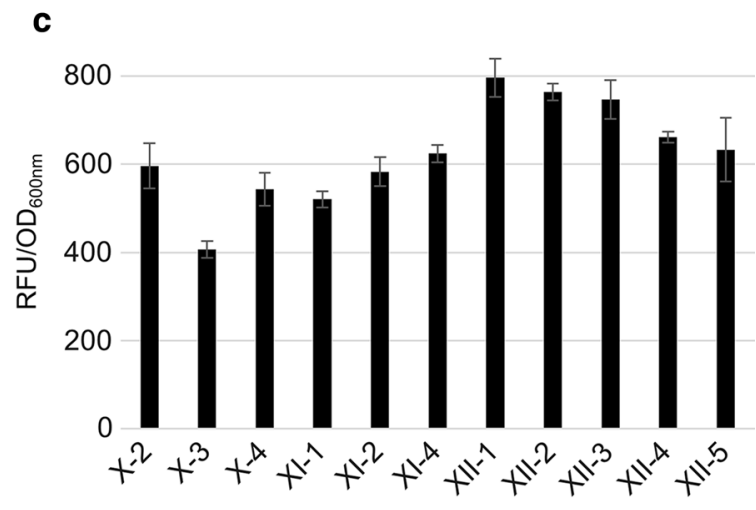

site integration. The specific fluorescence values were comparable between the vectors, though some integration sites gave slightly lower values (insertion site $\mathrm{X}-2$ ) and some slightly higher (insertion site XII-1) (Fig. 2c). This demonstrates the sufficient, homogenous and reliable level of expression, which the integration into these chromosomal locations mediated by the new vector set ensures.

To evaluate integration of prepared vectors and expression of cloned gene(s) in different industrial strains, three reporter genes encoding fluorescent proteins CFP, RFP and YFP under control of TEF1 promoter were cloned into the selected EasyClone vectors carrying different dominant markers, targeting insertion sites located at the same chromosome as well as the sites located at three different chromosomes (X, XI, XII) (Supplementary material Table S1). Such vectors were subsequently transformed into the laboratory CEN.PK and industrial Ethanol Red, CBS7960 and CLIB382 strains. All vectors could be introduced simultaneously into the CEN.PK strain, while in Ethanol Red and CLIB382 strains just two vector transformation yielded successful transformants. A second transformation was performed to introduce the third vector. In case of CBS7960 strain, reporter genes carrying vectors had to be introduced sequentially (Fig. 3a). As shown by fluorescence microscopy, the cells of each strain indeed expressed all three fluorescent proteins at homogenous level (Fig. 3b), independent of the combination of the vectors used (not shown).

\section{Selection markers can be removed by Cre-loxP recombination}

All the selection markers are flanked by loxP sites (Fig. 1a) allowing marker rescue mediated by CreA site-specific recombinase [13]. A one-step marker loop-out was performed in all tested strains by transforming the cells with a Cre-recombinase containing plasmid and subsequent induction of Cre expression. The insertion sites are interspaced by essential genetic elements, which prevents loopout of the inserted genes in haploid laboratory strains [26]. This advantage of the platform is naturally lost when used in di-/polyploid yeast. Therefore, there is a risk of inserted fragment loop-out caused by Cre-recombinase when neighboring insertion sites are used, encountering relatively close distance of several loxP sites. Indeed, a loss of at least one of the inserted reporter genes could be observed after Cre-mediated marker loop-out in case of industrial strains carrying the reporter genes on the same chromosome, while 

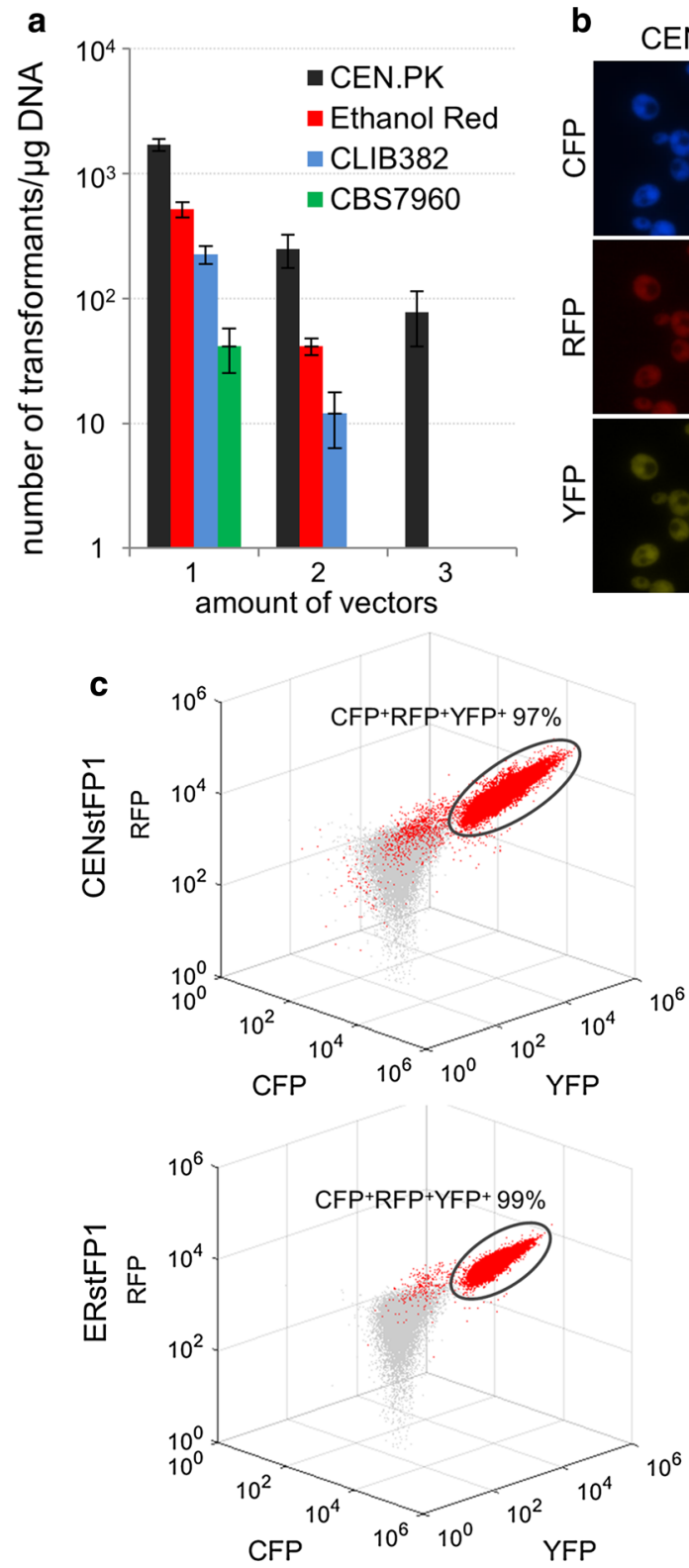
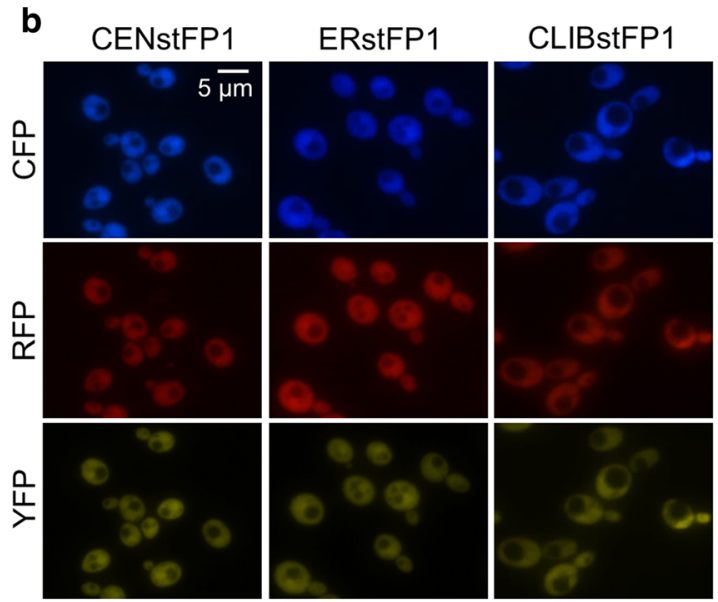

CBSstFP1
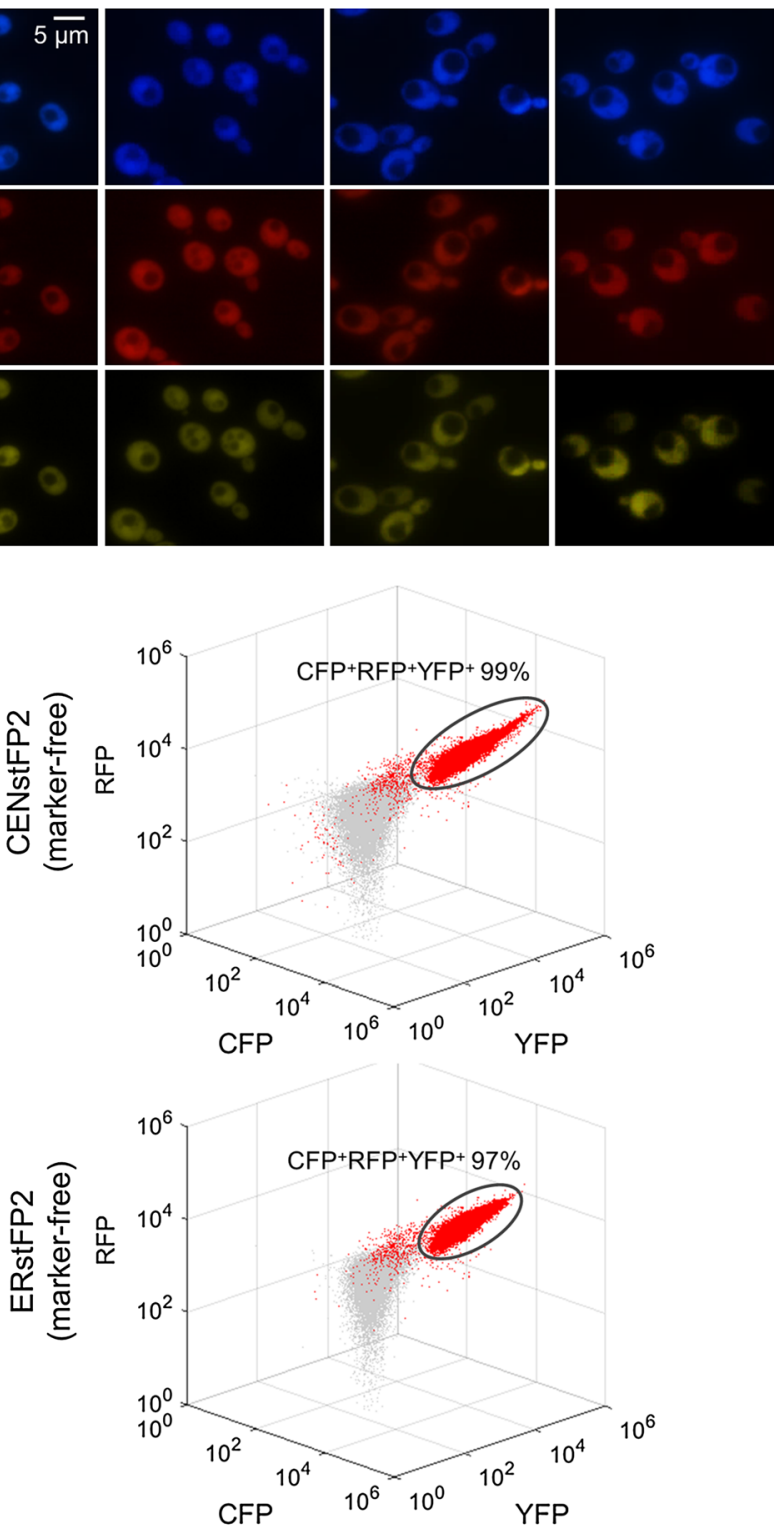

d

\begin{tabular}{|c|c|c|c|c|c|c|c|}
\hline Strain & $\begin{array}{c}\text { CFP+RFP+ } \\
\text { YFP+ }\end{array}$ & Strain & $\begin{array}{c}\text { CFP+RFP+ } \\
\text { YFP+ }\end{array}$ & Strain & $\begin{array}{c}\text { CFP+RFP+ } \\
\text { YFP+ }\end{array}$ & Strain & $\begin{array}{c}\text { CFP+RFP+ } \\
\text { YFP+ }\end{array}$ \\
\hline CENstFP $1 \mathrm{ev}^{*}$ & 96 & ERstFP $1 \mathrm{ev}^{*}$ & 98 & CBSstFP1 & 93 & CLIBstFP1 & 93 \\
\hline \multirow[t]{3}{*}{ CENstFP2 $2^{\mathrm{ev}^{*}}$} & 99 & ERstFP2 ${ }^{\mathrm{ev}^{*}}$ & 95 & CBSstFP1 $\mathrm{ev}^{*}$ & 93 & CLIBstFP $1 \mathrm{ev}^{*}$ & 93 \\
\hline & & ERstFP3 & 98 & & & & \\
\hline & & ERstFP $3 \mathrm{ev}^{*}$ & 98 & & & & \\
\hline
\end{tabular}

* ev - evolved strains grown for 35 generations under non-selective conditions

expectedly, there was no loss of the inserted genes in haploid CEN.PK strain. However, when the reporter genes were inserted at different chromosomal locations, a onestep marker removal was possible in diploid Ethanol Red strain without loss of the inserted genes. Flow cytometer analysis of the cell population did not reveal any significant changes of the populations in terms of the reporter gene fluorescence when comparing marker-containing and marker-free strains (Fig. 3c). To test the stability of the inserted genes, all reporter gene containing strains were 
4 Fig. 3 Integration of reporter genes delivered by the EasyClone 2.0 vectors in industrial yeast. a Transformation efficiency of the strains when transformed with 1,2 or 3 vectors simultaneously. Values of the transformation efficiency (expressed as a number of transformants per $\mu \mathrm{g}$ DNA) are plotted on logarithmic scale. The values represent an average from two independent experiments. Error bars represent standard deviation $(N=2)$. The amount of $10^{8}$ cells was used for each transformation. b Cells of the laboratory (CENstFP1) and industrial (ERstFP1, CBSstFP1, CLIBstFP1) strains expressing three different fluorescent proteins-CFP, RFP, YFP. c Flow cytometry analysis of the fluorescent protein expressing strains CENstFP1 (marker-containing), CENstFP2 (marker-free), ERstFP1 (markercontaining), ERstFP2 (marker-free). Three-dimensional scatter plots demonstrate the population properties of the fluorescent proteinexpressing strains (red populations). The values were plotted using scatter 3 function in MATLAB. Wild type (fluorescence negative) control overlay (grey populations) is given for comparison. The percentages of $\mathrm{CFP}^{+} \mathrm{YFP}^{+} \mathrm{RFP}^{+}$populations (delineated by the ellipse), as obtained by sequential gating in FlowJo (Supplementary material Fig. S2), are given. Two-dimensional contour plots of such populations are shown in Supplementary material Fig. S2. d The table displays the percentages of $\mathrm{CFP}^{+} \mathrm{YFP}^{+} \mathrm{RFP}^{+}$populations of the reporter gene expressing strains and their derivatives grown for 35 generations under non-selective conditions. The populations were analyzed in FlowJo as described in Supplementary material Fig. S2. Two-dimensional contour plots of such populations are shown in Supplementary material Fig. S2

grown for 35 generations under non-selective conditions. The resulting populations (including also several variants of industrial Ethanol Red, i.e., with the reporters on single chromosome, with the reporters on different chromosomes, both marker-containing and marker-free) were analyzed by flow cytometry. This analysis did not reveal any loss of the reporter genes in any strain or other significant differences between parental and derived strains. In CEN.PK and Ethanol Red strain derivatives, the vast majority of cells (more than $95 \%$ ) exhibited a homogenous level of reporter gene fluorescence (Fig. 3d, Supplementary material Fig. $\mathrm{S} 2$ ). Only a small part of the cell population of industrial CBS7960 and CLIB382 derivatives (around $7 \%$ ) showed decrease in fluorescence of the reporters. However, this was characteristic also for the parental population and not affected by the prolonged growth in selection-free conditions (Fig. 3d, Supplementary material Fig. S2). This demonstrated that the constructed EasyClone 2.0 vector set is suitable for stable heterologous gene insertions in industrial yeast and that dominant markers can be recycled using the Cre/loxP system.

\section{Construction of xylose utilizing strains}

The development of industrial xylose-fermenting yeast is of a great importance for second and third-generation biorefineries utilizing lignocellulosic feedstocks [22]. Two pathways for xylose utilization that have been mostly studied are oxidoreductase pathway, involving the expression of xylose reductase and xylitol dehydrogenase [18], and the xylose isomerase pathway, involving expression of heterologous xylose isomerase enzyme and xylulokinase [5]. The latter pathway does not have a cofactor imbalance issue and provides higher yields under industrially relevant anaerobic conditions $[6,21]$. We decided to construct xylose consuming strains with different industrial backgrounds as a case study, demonstrating the use of our integrative vector toolbox. To perform this, a strategy using the introduction of the xylose isomerase pathway and tuning of pentose phosphate pathway was selected (Fig. 4a). The crucial step is to ensure sufficient activity of heterologous xylose isomerase. To be able to introduce more copies of a gene, we constructed multiple integration vectors (Maury et al., unpublished results), targeting LTR sequences of yeast Ty2 and Ty4 transposable elements spread in yeast genome in several copies, allowing for selection in prototrophic yeast (Supplementary material Table S1). The vector with Ty2 integration site was used for cloning of the XylA gene from Clostridium phytofermentans [5] along with the P. stipitis XYL3 gene encoding D-xylulokinase. Selected single integration vectors were used for cloning of the P. stipitis SUT1 gene (along with a $C p X y l A$ allele), encoding a sugar transporter with higher affinity for xylose, and 4 genes of the non-oxidative part of the pentose phosphate pathway (PPP): three native $S$. cerevisiae genes (RKI1, RPE1, TKL1) and transaldolase from P. stipitis (PsTAL1) (Fig. 4b). We chose the PsTAL1 gene because it has been shown previously that its overexpression does not influence the growth rate, in contrast to the native TAL1 allele [19]. The genes were cloned under control of the strong constitutive promoters $P_{T E F 1}$ and $P_{T D H 3}$ (Fig. 4b). The vectors were successfully inserted into the genomes of laboratory CEN.PK and industrial Ethanol Red and CLIB382 strains, chosen to represent two different industrial backgrounds. The insertion of three vectors was achieved in a single transformation for laboratory strain and in two transformation rounds for industrial strains. Finally, the resulting strains were transformed with Ty2 vector carrying CpXylA and PsXYL3 genes. To select for multiple integration, the concentration of selective antibiotic was increased 5-fold, and xylose containing complex medium was used. The resulting transformants were screened for growth in YPX (and YPD for comparison) liquid medium using microplate reader. The fastest growing isolates (Fig. 4c) were evaluated in time course experiment in shake flasks. Furthermore, $C p X y l A$ gene copy number of such strains was determined by qPCR. CENstXYL (containing 6 copies of $C p X y l A$ gene), ERstXYL (8 copies of $C p X y l A$ ) and CLIBstXYL (8 copies of $C p X y l A)$ strains were grown for $60 \mathrm{~h}$ under aerobic conditions in complex YPX medium and the xylose concentration and the maximum specific growth rates $\mu_{\max }$ were determined. CENstXYL strain exhibited the fastest growth 

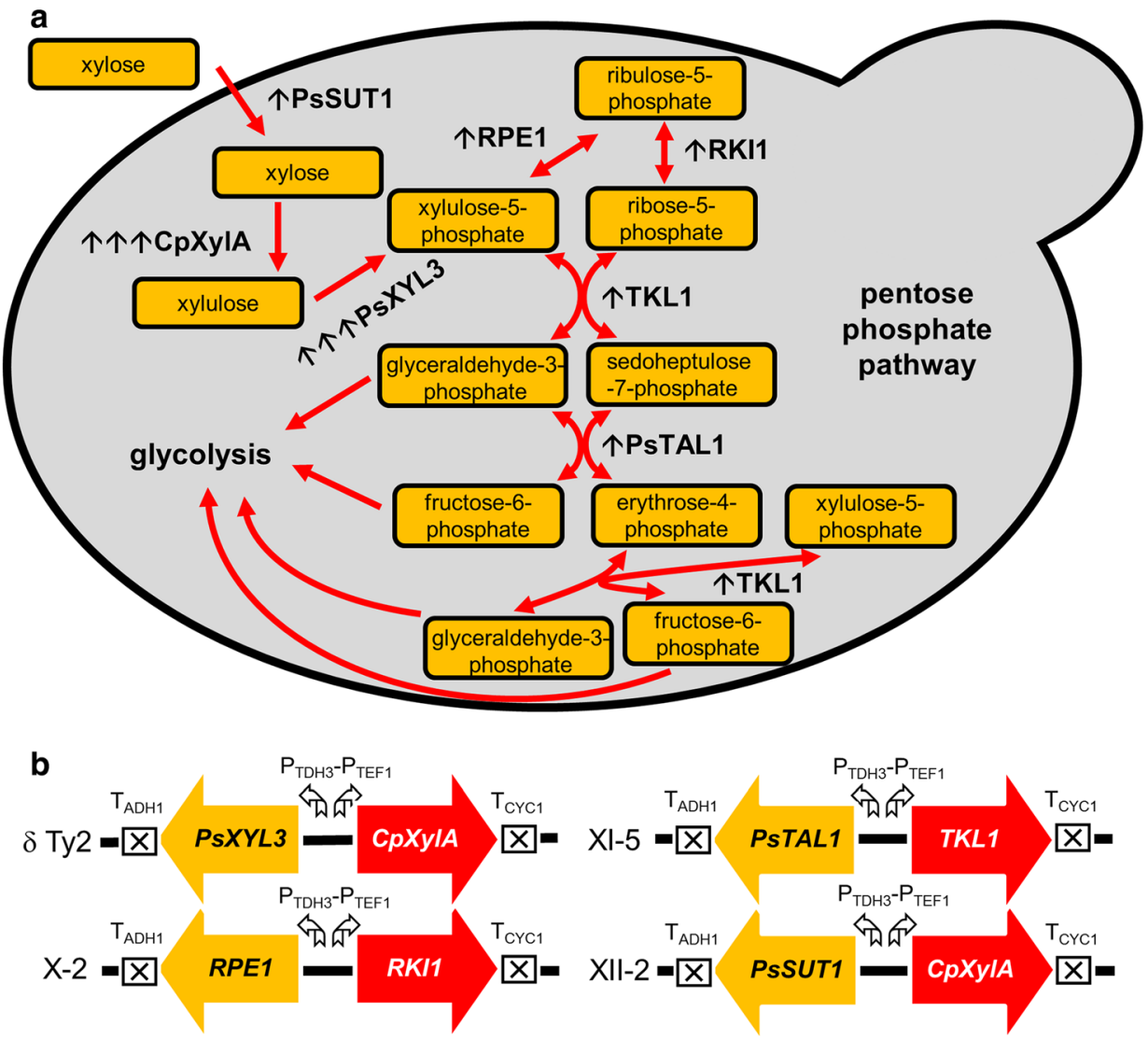

C

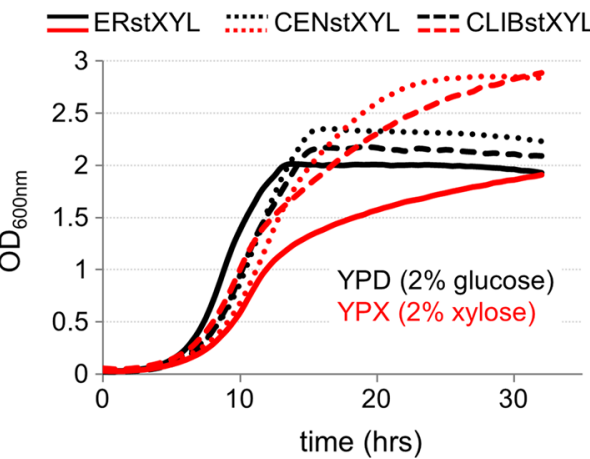

Fig. 4 Engineering of industrial strains for xylose utilization. a Schematic illustration of the xylose isomerase pathway. The genes integrated using the EasyClone2.0 vectors are displayed. Single black arrows $(\uparrow)$ represent single integration, triple arrows $(\uparrow \uparrow \uparrow)$ represent multiple integration. b Schematic illustration of chromosomal insertions of the cloned xylose pathway genes in the genomes of CENstXYL, ERstXYL and CLIBstXYL strains. Particular chromosomal locations are displayed as well as promoters and terminators. c Growth curves of CENstXYL, ERstXYL and CLIBstXYL strains. The strains were grown in $100 \mu 1$ of YPD (black curves) and YPX (red curves) media in microtiter plates, OD was determined with Biotek ELx808 microplate reader in 30 min intervals. The values

$\left(\mu_{\max }=0.296 \mathrm{~h}^{-1}\right)$ and reached the highest OD (approximately after $24 \mathrm{~h}$ of growth), CLIBstXYL strain showed similar parameters $\left(\mu_{\max }=0.283 \mathrm{~h}^{-1}\right)$ though reaching d

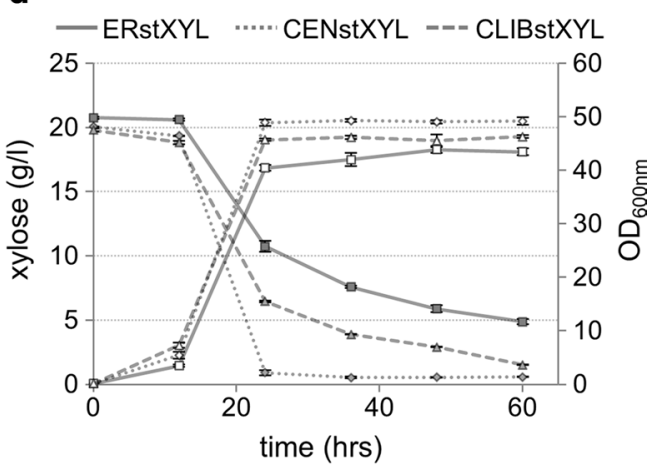

were normalized to a $1 \mathrm{~cm}$ pathlength using pathlength correction performed according to manufacturer's instructions. $\mathbf{d}$ The graph represents time course of xylose consumption by CENstXYL, ERstXYL and CLIBstXYL strains grown in $50 \mathrm{ml}$ YPX medium in shake flasks. Samples were taken at regular time points and OD of the culture was determined with the NanoPhotometer Pearl (Implen, Germany) in a $1 \mathrm{~cm}$ pathlength cuvette, and xylose concentration was measured by HPLC. Xylose concentration is plotted on the primary $y$ axis (closed symbols), OD values (open symbols) on the secondary y axis. The cultures were cultivated in triplicates. Error bars represent standard deviation $(N=3)$

slightly lower OD, while ERstXYL strain grew slower $\left(\mu_{\max }=0.271 \mathrm{~h}^{-1}\right.$, Supplementary material Fig. S3). CENstXYL strains consumed the entire xylose content in 
$24 \mathrm{~h}$, and CLIBstXYL strains in $60 \mathrm{~h}$. In ERstXYL cultivation, $75 \%$ of the xylose content was consumed after 60 h (Fig. 4d).

\section{Discussion}

Stress-tolerant and robust industrial yeast strains are suitable production organisms in large-scale industrial biorefineries, where biomass feedstocks are fermented into fuels and chemicals [32]. Heterologous gene insertions and their controlled expression are crucial events needed for the introduction of new cellular processes enabling production of non-natural products and optimization of the desired cellular properties. A common feature for genetic manipulations is the requirement of genetic markers that enable the selection of mutants [25]. Engineering of prototrophic industrial yeast strains prevents the use of auxotrophic selection and requires use of dominant markers [2]. The presented EasyClone 2.0 set of integrative vectors allow for gene insertions and selection in prototrophic yeast strains. The substances used as selection agents do not show any cross-reactivity and can be used as selective agents simultaneously. This is especially important when more genes are considered to be introduced. Previously presented vector sets with dominant markers do not provide such broad option of marker selection and/or provide only limited options of promoter choice to regulate the expression of an inserted gene [23, 47]. The EasyClone vectors enable free choice of any promoter to control the expression [17]. Level of expression, its homogeneity and reliability are an important consideration of any industrial process design. This is an issue when episomal vectors are used, since the homogenous expression cannot be controlled [17, 42]. Although possible to use in prototrophic strains, e.g., for a gene expression evaluation [49], such vectors are not suitable for construction of efficient cell factories. The constructed second generation of EasyClone vectors, designed to integrate into previously validated integration site on three different $S$. cerevisiae chromosomes [26], provides sufficient and mutually comparable level of expression.

Industrial strains often display low accessibility in terms of foreign DNA take up [44]. The vector set is equipped with long insertion sequences. This provides universality of such vectors as shown by the successful insertion and expression of three different reporter genes in unrelated strains, despite originally designed for laboratory strains [26]. This is an apparent advantage when compared to previously constructed vector sets integrating at also well-defined chromosomal locations, using however PCR-generated short insertion sequences [9]. Short homologous sequences might cause lower probability of successful insertion and subsequent lower yield of correct transformants. Furthermore, sufficiently long insertion regions allowed introduction of several vectors simultaneously in the tested strains, except the CBS7960, which represents a hardly accessible strain in general [46]. Besides the homogenous and controlled level of expression, the stability of the inserted genetic material is another important consideration. Diploid industrial strains lack the advantage of the expression platform interspaced by essential genes, preventing homologous recombination-mediated gene loss [26]. Despite this, the presented set enables stable integration expression of heterologous genes after prolonged cultivation under non-selective conditions. Even when genes are inserted on the same chromosome, no loss of the inserted reporters was observed.

Some previously reported dominant marker containing vectors lack marker excision possibility and thus prevent repeated use of the vectors and markers [47]. Moreover, the final production strains should not contain antibiotic resistance markers to avoid rise of antibiotics resistance among pathogens via horizontal gene transfer. The presented set carry markers flanked with loxP sites allowing for one-step marker rescue [13], being widely used marker recycling strategy [40]. The successful one-step marker excision was demonstrated. However, when the genes were inserted on the same chromosome, Cre-mediated marker loop-out was accompanied also by the loss of a reporter gene in diploid strains. This happened most likely due to accumulation of six loxP sites in relatively short, $42 \mathrm{kbp}$, distance [17]. Thus, one must pay special attention to a selection of suitable vectors and planning of simultaneous and sequential integrations to avoid such situation when excision of the markers in diploid strains is intended.

For the development of third-generation biorefineries, producing valuable chemicals from non-food biomass such as lignocellulosic substrates, conversion of C5-rich streams is an important cost-reductive challenge. For this reason, many studies have faced this challenge and succeeded in engineering of even industrial $S$. cerevisiae strains, naturally not fermenting $\mathrm{C} 5$ streams, for utilization of $\mathrm{C} 5 \mathrm{sug}$ ars [37-39]. Based on great importance of C5-fermentation in $S$. cerevisiae, we constructed xylose consuming strains as a valuable proof-of-concept of applicability of the constructed vector set in metabolic engineering of industrial strains. To ensure sufficiently high expression (activity) of xylose isomerase, we enriched our vector set for vectors targeting $\delta$ sequences of Ty 2 transposons (Maury et al., unpublished results). These elements are spread in several copies in yeast genome and enable insertion of a gene in many copies [39]. In contrast to the stable nature of singlesite genome integrations, certain stability issues of multiple copy $\delta$-based insertions have been documented [51]. However, our strategy considered the presence of xylose acting as natural selection pressure, preventing the potential 
risk of xylose isomerase gene loss. Moreover, the use of such insertion sites might facilitate further multiplication of XylA copy number, an event responsible for improved xylose metabolism after long-term evolution in the presence of xylose [7]. The engineered strains were able to utilize xylose as sole carbon source, with different properties dependent apparently on strain background. Further optimizing strategies, e.g., knock-out of GRE3 to avoid production of xylitol as an inhibitor of xylose isomerase activity [21] and adaptive laboratory evolution [6] will be pursued in the future work to improve the xylose metabolism.

Taken together, we constructed the second generation of integrative vectors which mediate (heterologous) gene expression in industrial yeast (overview of EasyClone 2.0 in Supplementary material Fig. S4). The vectors provide (1) a wide range of dominant marker options for selection in (prototrophic) yeast strains, (2) free choice of a promoter and homogenous level of expression, (3) universality for use in strains with different genetic background, (4) stability of the inserted genes and (5) marker excision and recycling possibilities for repeated rounds of gene insertions. Our results also document the applicability of the vector set for metabolic engineering of industrial strains. The presented toolbox significantly broadens the options of design and engineering of industrial cell factories.

Acknowledgments This work is part of BioREFINE-2G project, which is co-funded by the European Commission in the 7th Framework Programme (Project No. FP7-613771). The authors also acknowledge the Novo Nordisk Foundation for financial support. The authors thank Laura Dato for help with FACS analysis and Tomas Strucko for help with MATLAB script.

Open Access This article is distributed under the terms of the Creative Commons Attribution 4.0 International License (http://creativecommons.org/licenses/by/4.0/), which permits unrestricted use, distribution, and reproduction in any medium, provided you give appropriate credit to the original author(s) and the source, provide a link to the Creative Commons license, and indicate if changes were made.

\section{References}

1. Attfield PV (1997) Stress tolerance: the key to effective strains of industrial baker's yeast. Nat Biotechnol 15:1351-1357. doi:10.1038/nbt1297-1351

2. Le Borgne S (2012) Genetic engineering of industrial strains of Saccharomyces cerevisiae. Methods Mol Biol Clifton NJ 824:451-465. doi:10.1007/978-1-61779-433-9_24

3. Borodina I, Jensen BM, Søndergaard I, Poulsen LK, Borodina I, Jensen BM, Søndergaard I, Poulsen LK (2010) Display of wasp venom allergens on the cell surface of Saccharomyces cerevisiae. Microb Cell Fact. doi:10.1186/1475-2859-9-74

4. Borodina I, Nielsen J (2014) Advances in metabolic engineering of yeast Saccharomyces cerevisiae for production of chemicals. Biotechnol J 9:609-620. doi:10.1002/biot.201300445

5. Brat D, Boles E, Wiedemann B (2009) Functional expression of a bacterial xylose isomerase in Saccharomyces cerevisiae. Appl Environ Microbiol 75:2304-2311. doi:10.1128/AEM.02522-08
6. Demeke MM, Dietz H, Li Y, Foulquié-Moreno MR, Mutturi S, Deprez S, Abt TD, Bonini BM, Liden G, Dumortier F, Verplaetse A, Boles E, Thevelein JM (2013) Development of a D-xylose fermenting and inhibitor tolerant industrial Saccharomyces cerevisiae strain with high performance in lignocellulose hydrolysates using metabolic and evolutionary engineering. Biotechnol Biofuels 6:89. doi:10.1186/1754-6834-6-89

7. Demeke MM, Foulquié-Moreno MR, Dumortier F, Thevelein JM (2015) Rapid evolution of recombinant Saccharomyces cerevisiae for Xylose fermentation through formation of extra-chromosomal circular DNA. PLoS Genet 11:e1005010. doi:10.1371/ journal.pgen. 1005010

8. Diao L, Liu Y, Qian F, Yang J, Jiang Y, Yang S (2013) Construction of fast xylose-fermenting yeast based on industrial ethanol-producing diploid Saccharomyces cerevisiae by rational design and adaptive evolution. BMC Biotechnol 13:110. doi:10.1186/1472-6750-13-110

9. Fang F, Salmon K, Shen MWY, Aeling KA, Ito E, Irwin B, Tran UPC, Hatfield GW, Da Silva NA, Sandmeyer S (2011) A vector set for systematic metabolic engineering in Saccharomyces cerevisiae. Yeast Chichester Engl 28:123-136. doi:10.1002/ yea.1824

10. Flagfeldt DB, Siewers V, Huang L, Nielsen J (2009) Characterization of chromosomal integration sites for heterologous gene expression in Saccharomyces cerevisiae. Yeast Chichester Engl 26:545-551. doi:10.1002/yea.1705

11. Futcher AB, Cox BS (1983) Maintenance of the 2 microns circle plasmid in populations of Saccharomyces cerevisiae. J Bacteriol 154:612-622

12. Gietz RD, Woods RA (2006) Yeast transformation by the LiAc/ SS Carrier DNA/PEG method. Methods Mol Biol Clifton NJ 313:107-120. doi:10.1385/1-59259-958-3:107

13. Guldener U, Heck S, Fielder T, Beinhauer J, Hegemann JH (1996) A new efficient gene disruption cassette for repeated use in budding yeast. Nucleic Acids Res 24:2519-2524

14. Hahn-Hägerdal B, Karhumaa K, Jeppsson M, Gorwa-Grauslund MF (2007) Metabolic engineering for pentose utilization in Saccharomyces cerevisiae. Adv Biochem Eng Biotechnol 108:147177. doi:10.1007/10_2007_062

15. Ishii J, Kondo T, Makino H, Ogura A, Matsuda F, Kondo A (2014) Three gene expression vector sets for concurrently expressing multiple genes in Saccharomyces cerevisiae. FEMS Yeast Res 14:399-411. doi:10.1111/1567-1364.12138

16. Jakočiunas T, Rajkumar AS, Zhang J, Arsovska D, Rodriguez A, Jendresen CB, Skjødt ML, Nielsen AT, Borodina I, Jensen MK, Keasling JD (2015) CasEMBLR: Cas9-facilitated Multiloci genomic integration of in vivo assembled DNA parts in Saccharomyces cerevisiae. ACS Synth Biol. doi:10.1021/acssynbio.5b00007

17. Jensen NB, Strucko T, Kildegaard KR, David F, Maury J, Mortensen UH, Forster J, Nielsen J, Borodina I (2014) EasyClone: method for iterative chromosomal integration of multiple genes in Saccharomyces cerevisiae. FEMS Yeast Res 14:238248. doi:10.1111/1567-1364.12118

18. Jeppsson M, Bengtsson O, Franke K, Lee H, Hahn-Hägerdal B, Gorwa-Grauslund MF (2006) The expression of a Pichia stipitis xylose reductase mutant with higher $\mathrm{K}(\mathrm{M})$ for NADPH increases ethanol production from xylose in recombinant Saccharomyces cerevisiae. Biotechnol Bioeng 93:665-673. doi:10.1002/ bit.20737

19. Jin Y-S, Alper H, Yang Y-T, Stephanopoulos G (2005) Improvement of xylose uptake and ethanol production in recombinant Saccharomyces cerevisiae through an inverse metabolic engineering approach. Appl Environ Microbiol 71:8249-8256. doi:10.1128/AEM.71.12.8249-8256.2005

20. Katz M, Durhuus T, Smits HP, Förster J (2011) Production of metabolites. Patent WO2011147818 
21. Kuyper M, Hartog MMP, Toirkens MJ, Almering MJH, Winkler AA, van Dijken JP, Pronk JT (2005) Metabolic engineering of a xylose-isomerase-expressing Saccharomyces cerevisiae strain for rapid anaerobic xylose fermentation. FEMS Yeast Res 5:399409. doi:10.1016/j.femsyr.2004.09.010

22. Lee J (1997) Biological conversion of lignocellulosic biomass to ethanol. J Biotechnol 56:1-24. doi:10.1016/ S0168-1656(97)00073-4

23. Leite FCB, Dos Anjos RSG, Basilio ACM, Leal GFC, Simões DA, de Morais MA (2013) Construction of integrative plasmids suitable for genetic modification of industrial strains of Saccharomyces cerevisiae. Plasmid 69:114-117. doi:10.1016/j. plasmid.2012.09.004

24. Li M, Borodina I (2014) Application of synthetic biology for production of chemicals in yeast $S$. cerevisiae. FEMS. Yeast. doi:10.1111/1567-1364.12213

25. Mapelli V (2014) Yeast metabolic engineering. Springer, New York

26. Mikkelsen MD, Buron LD, Salomonsen B, Olsen CE, Hansen BG, Mortensen UH, Halkier BA (2012) Microbial production of indolylglucosinolate through engineering of a multi-gene pathway in a versatile yeast expression platform. Metab Eng 14:104111. doi:10.1016/j.ymben.2012.01.006

27. Nevoigt E (2008) Progress in metabolic engineering of Saccharomyces cerevisiae. Microbiol Mol Biol Rev MMBR 72:379412. doi:10.1128/MMBR.00025-07

28. Nielsen J (2013) Production of biopharmaceutical proteins by yeast. Bioengineered 4:207-211. doi:10.4161/bioe.22856

29. Nielsen J, Larsson C, van Maris A, Pronk J (2013) Metabolic engineering of yeast for production of fuels and chemicals. Curr Opin Biotechnol 24:398-404. doi:10.1016/j.copbio.2013.03.023

30. Nørholm MH (2010) A mutant Pfu DNA polymerase designed for advanced uracil-excision DNA engineering. BMC Biotechnol 10:21. doi:10.1186/1472-6750-10-21

31. Nour-Eldin HH, Geu-Flores F, Halkier BA (2010) USER cloning and USER fusion: the ideal cloning techniques for small and big laboratories. Methods Mol Biol Clifton NJ 643:185-200. doi:10.1007/978-1-60761-723-5_13

32. Paes BG, Almeida JR (2014) Genetic improvement of microorganisms for applications in biorefineries. Chem Biol Technol Agric 1:21. doi:10.1186/s40538-014-0021-1

33. Parreiras LS, Breuer RJ, Avanasi Narasimhan R, Higbee AJ, La Reau A, Tremaine M, Qin L, Willis LB, Bice BD, Bonfert BL, Pinhancos RC, Balloon AJ, Uppugundla N, Liu T, Li C, Tanjore D, Ong IM, Li H, Pohlmann EL, Serate J, Withers ST, Simmons BA, Hodge DB, Westphall MS, Coon JJ, Dale BE, Balan V, Keating DH, Zhang Y, Landick R, Gasch AP, Sato TK (2014) Engineering and two-stage evolution of a lignocellulosic hydrolysate-tolerant Saccharomyces cerevisiae strain for anaerobic fermentation of xylose from AFEX pretreated corn stover. PLoS One 9:e107499. doi:10.1371/journal.pone.0107499

34. Partow S, Siewers V, Bjørn S, Nielsen J, Maury J (2010) Characterization of different promoters for designing a new expression vector in Saccharomyces cerevisiae. Yeast Chichester Engl 27:955-964. doi:10.1002/yea.1806

35. Pereira FB, Guimarães PMR, Teixeira JA, Domingues L (2010) Selection of Saccharomyces cerevisiae strains for efficient very high gravity bio-ethanol fermentation processes. Biotechnol Lett 32:1655-1661. doi:10.1007/s10529-010-0330-9
36. Reid RJD, Sunjevaric I, Keddache M, Rothstein R, Kedacche M (2002) Efficient PCR-based gene disruption in Saccharomyces strains using intergenic primers. Yeast Chichester Engl 19:319328. doi:10.1002/yea.817

37. Ryan OW, Skerker JM, Maurer MJ, Li X, Tsai JC, Poddar S, Lee ME, DeLoache W, Dueber JE, Arkin AP, Cate JH (2014) Selection of chromosomal DNA libraries using a multiplex CRISPR system. ELife. doi:10.7554/eLife.03703

38. Sadowski I, Su T-C, Parent J (2007) Disintegrator vectors for single-copy yeast chromosomal integration. Yeast Chichester Engl 24:447-455. doi:10.1002/yea.1469

39. Sakai A, Shimizu Y, Hishinuma F (1990) Integration of heterologous genes into the chromosome of Saccharomyces cerevisiae using a delta sequence of yeast retrotransposon Ty. Appl Microbiol Biotechnol 33:302-306. doi:10.1007/BF00164526

40. Sauer B (1994) Recycling selectable markers in yeast. Biotechniques 16:1086-1088

41. Shao Z, Zhao H, Zhao H (2009) DNA assembler, an in vivo genetic method for rapid construction of biochemical pathways. Nucleic Acids Res 37:e16. doi:10.1093/nar/gkn991

42. Da Silva NA, Srikrishnan S (2012) Introduction and expression of genes for metabolic engineering applications in Saccharomyces cerevisiae. FEMS Yeast Res 12:197-214. doi:10.1111/j.1567-1364.2011.00769.x

43. Solis-Escalante D, Kuijpers NGA, Bongaerts N, Bolat I, Bosman L, Pronk JT, Daran J-M, Daran-Lapujade P (2013) amdSYM, a new dominant recyclable marker cassette for Saccharomyces cerevisiae. FEMS Yeast Res 13:126-139. doi:10.1111/1567-1364.12024

44. Spencer JFT, Spencer DM (1983) Genetic improvement of industrial yeasts. Annu Rev Microbiol 37:121-142. doi:10.1146/ annurev.mi.37.100183.001005

45. Stearns T, Ma H, Botstein D (1990) Manipulating yeast genome using plasmid vectors. Methods Enzymol 185:280-297

46. Stovicek V, Borodina I, Forster J (2015) CRISPR-Cas system enables fast and simple genome editing of industrial Saccharomyces cerevisiae strains. Metab Eng Commun 2:13-22. doi:10.1016/j.meteno.2015.03.001

47. Taxis C, Knop M (2006) System of centromeric, episomal, and integrative vectors based on drug resistance markers for Saccharomyces cerevisiae. Biotechniques 40:73-78

48. Verwaal R, Wang J, Meijnen J-P, Visser H, Sandmann G, van den Berg JA, van Ooyen AJJ (2007) High-level production of betacarotene in Saccharomyces cerevisiae by successive transformation with carotenogenic genes from. Appl Environ Microbiol 73:4342-4350. doi:10.1128/AEM.02759-06

49. Vickers CE, Bydder SF, Zhou Y, Nielsen LK (2013) Dual gene expression cassette vectors with antibiotic selection markers for engineering in Saccharomyces cerevisiae. Microb Cell Fact 12:96. doi:10.1186/1475-2859-12-96

50. Vorachek-Warren MK, McCusker JH (2004) DsdA (D-serine deaminase): a new heterologous MX cassette for gene disruption and selection in Saccharomyces cerevisiae. Yeast Chichester Engl 21:163-171. doi:10.1002/yea.1074

51. Wang X, Wang Z, Da Silva NA (1996) G418 Selection and stability of cloned genes integrated at chromosomal delta sequences of Saccharomyces cerevisiae. Biotechnol Bioeng 49:45-51. doi:10.1002/ (SICI)1097-0290(19960105)49:1<45:AID-BIT6>3.0.CO;2-T 